\title{
Evaluation of time-varying working posture based on interjoint coordination features extracted from sparse structure learning
}

\author{
Kazuki HIRANAI* and Akihiko SEO** \\ *Graduate School of Systems Design, Tokyo Metropolitan University \\ 6-6 Asahigaokai, Hino, Tokyo 191-0065, Japan \\ E-mail: hiranai-kazuki@ed.tmu.ac.jp \\ **Faculty of Systems Design, Tokyo Metropolitan University \\ 6-6 Asahigaokai, Hino, Tokyo 191-0065, Japan
}

Received: 26 November 2020; Revised: 2 January 2021; Accepted: 1 February 2021

\begin{abstract}
To evaluate tasks that have low physical workloads or small variations in the working postures, a method that can be used to analyze slight differences in postures and movements based on human motion characteristics is required. The interjoint coordination, which produces multijoint movements by organizing the redundant degrees of freedom into fewer major covarying relationships, is one of the primary characteristics of human motion. This work proposes a novel evaluation method for interjoint coordination using the graphical lasso and clarifies its efficacy for evaluations of interjoint coordination in complete tasks as well as time-varying interjoint coordination and working postures. In a subject experiment, eleven male participants performed lightweight material-handling tasks under different working conditions and paces, and an electromagnetic motion-tracking system was used to measure their working postures. The principal interjoint coordination for measured joint angles was extracted using the graphical lasso tool as a sparse precision matrix. Further, the contribution of each joint angle to the changes in the working postures in the time series was calculated by correlation anomaly using the graphical lasso. The estimated sparse precision matrix using the graphical lasso suggested that the principal interjoint coordination reflecting the differences in movement strategies by task type can be extracted in comparison with the covariance matrix. The time-varying correlation anomaly score suggested that the joint angle that contributes to differences between interjoint coordination at the onset and termination of tasks could be determined according to the final score. Therefore, our study shows the efficacy of the graphical lasso for evaluation of interjoint coordination and working postures from the time series for repetitive lightweight material-handling tasks.
\end{abstract}

Keywords : Anomaly detection, Feature extraction, Sparse structure learning, Interjoint coordination, Repetitive task, Human motion analysis, Working posture

\section{Introduction}

Manual work, which has a greater physical workload than other types of work, has been gradually reduced in the industry in recent times because of automation of the production process. However, most automation techniques still have several challenges, such as limited flexibility and greater introduction cost (Kadir et al., 2019). Therefore, complex tasks that require human skills for learning, adapting, or problem-solving are difficult to automate and depend on manual work by employees (Fantini et al., 2016; Forsman et al., 2002). To design a workstation that is comfortable for the employees, evaluations of working postures and physical workload are still considered important. Manual work in recent times mainly comprises tasks involving low physical workloads or small variations in the working postures; hence, a work evaluation method is required to detect and analyze slight differences in employee postures and movements. Observational methods and biomechanical analyses are representative ergonomic evaluation methods that 
are often used for work evaluations.

Observational methods, such as the Ovako working posture analysing system (OWAS) (Karhu et al., 1977) and the rapid upper limb assessment (RULA) (McAtamney and Corlett, 1993), evaluate the physical workloads in tasks based on visual determination of the working posture by an observer. However, as observational methods may be subject to intra- and interobserver variabilities when choosing between different categories of exposure levels, objective evaluations of working postures and physical workloads may be difficult. Biomechanical analyses, including digital human modeling, which simulates the human body using a simple multibody dynamics model, are often used to estimate the working postures and physical workloads (Chaffin et al., 2006). This analysis provides quantitative evaluations by calculating the angle and torque of each joint as well as their associated temporal changes. However, as recent motion capture technologies have the capability to acquire large amounts of data on human posture and motion, it is difficult to detect specific behaviors in working postures or motions. Therefore, feature extraction from human motion, which is often used in computer vision applications, is useful for detecting and analyzing slight differences in the working postures and motions based on movement features during tasks. The purpose of feature extraction is to analyze human motion effectively based on understanding changes in patterns. Previously, several feature-extraction methods have been proposed, such as segmentation of human motion (San-Segundo et al., 2016), signal transformation using wavelet transforms (Kadrolkar and Sup IV, 2017), and principal component analysis (PCA) (Masoud et al., 2017).

The human musculoskeletal system is a complex mechanical system with many degrees of freedom (DoFs), which allows flexible and adaptable movements. Ordinarily, humans achieve multijoint movements by organizing redundant DoFs into fewer major covarying relationships, thus indicating high-level interjoint coordination (Cowley and Gates, 2017). Interjoint coordination is one of the primary characteristics of human motion. Bernstein (1967) stated that when beginners first approach a novel movement strategy, they rigidly and spastically move their body to constrain the motor system and that the initial skill acquisition can therefore be characterized by freezing the kinematic DoFs. Cowley and Gates (2017) investigated the changes in interjoint coordination during muscle fatigue, which can be defined as a reduction in muscle capacity to generate force, thereby causing altered motor recruitment (Farina et al., 2008) and increased force and movement variabilities (Côté et al., 2008; Gates and Dingwell, 2011). Thus, movement strategies accounting for muscle fatigue may differ from those without muscle fatigue (Huffenus et al., 2006). Indeed, several studies have reported that during repetitive tasks, workers may compensate for muscle fatigue by reorganizing their movement patterns for the individual DoFs (McDonald et al., 2016) or by utilizing different DoFs, which alter the interjoint coordination.

One of the traditional evaluation methods for changes in the interjoint coordination is dependent on the average or maximum joint position during movement (Côté et al., 2002). However, this method cannot quantify how multiple joints coordinate to generate a movement. Owing to multivariate statistical analysis, such as factor analysis, nonnegative factorization, or PCA, the structure of interjoint coordination can be quantitatively evaluated. In particular, PCA is often used to quantify differences in multijoint movements across conditions to identify how different conditions affect movement strategies (Wang et al., 2013). Moreover, Varrel et al. (2013) reported that the magnitude of the interjoint cross-correlation in tasks represented the degree of interjoint coupling.

Sparse structure learning encodes a graphical structure that represents the intervariable relationship and learning this structure may be helpful for evaluating the changes in interjoint coordination during repetitive tasks. In statistics, the problem of structure learning has been explored within the framework of covariance selection (Dempster, 1972), wherein the multivariate Gaussian is assumed to be a generative model to fit a precision matrix that is the inverse of the covariance matrix to the data under a sparsity constraint. However, it is known that covariance selection has several limitations in practice, such as high computational cost and suboptimality in terms of the statistical tests. Moreover, by necessity, the covariance selection approach cannot be applied to data that do not exist in the inverse of the covariance matrix. In recent years, a graphical lasso that solves these problems has been proposed and has enabled the application of sparse structure learning to noisy real data (Friedman et al., 2008). The graphical lasso tool has been applied to predict traffic flows (Sun et al., 2012) and for image classification (Li et al., 2016).

The present study proposes a novel evaluation method for interjoint coordination using the graphical lasso and clarifies its efficacy for evaluating interjoint coordination in complete tasks as well as time-varying interjoint coordination and working postures. Specifically, the graphical lasso is applied to the measured joint angles in repetitive lightweight material-handling tasks, where the task types and paces change, to evaluate interjoint coordination. 
Moreover, the joint angle that most influences the time-varying interjoint coordination is estimated based on the correlation anomaly concept proposed by Ide et al. (2009).

\section{Theory}

\subsection{Graphical lasso}

Ide et al. (2009) assumed that if a system is working normally, the neighborhood graph of each node is almost invariant to changes in experimental conditions. In addition, they equated the problem of correlation anomaly detection to detecting significant changes in the neighborhood graphs of the variables involved. To represent the intervariable relationships, a simple model that focuses only on the relationship between two variables is often used. The edge between the two variables is absent if and only if they are independently conditioned for all the other variables. Based on the above condition, the neighborhood graph is constructed from the probability distribution. The learning problem for the neighborhood graph structure is redefined as that for the probability distribution from the measured data. The graph model, which assumes a multivariate normal distribution, is called the Gaussian graphical model. Structure learning on the Gaussian graphical model is defined as the problem of estimating the precision matrix, which is the inverse of the covariance matrix, and represents the direct correlations between variables. To focus on the principal relationships between the variables, it is necessary to estimate the sparse precision matrix.

Suppose that there are data $\mathcal{D}=\left\{\boldsymbol{x}^{(n)} \mid n=1, \cdots, N\right\}$ composed of $N$ multivariate normal observations of dimension $M$; we assume that $\mathcal{D}$ is standardized to have zero mean and unit variance. The sample covariance matrix of $\mathbf{S}_{i, j}$ is denoted as

$\mathbf{S}_{i, j} \equiv \frac{1}{N} \sum_{n=1}^{N} x_{i}^{(n)} x_{j}^{(n)}$.

Here, $\mathbf{S}_{i, j}$ is identical to the correlation coefficient matrix of $\mathcal{D}$ before standardization. The precision matrix $\boldsymbol{\Lambda}$ is the inverse of the covariance matrix $\mathbf{S}$ and presents the direct correlations between the variables. When the number of variables exceeds 10 , the covariance matrix is almost never the holomorphic matrix. Moreover, even if the covariance matrix is a holomorphic matrix, the precision matrix is almost never the sparse matrix. Therefore, the sparse precision matrix is estimated using a graphical lasso proposed by Friedman et al. (2008), which is a structure selection algorithm based on maximum likelihood estimation with $L_{1}$-penalized. Specifically, the sparse precision matrix is learned using the following procedure of the graphical lasso:

Initialization: Given the regularization coefficient, after data $\mathcal{D}$ is normalized, the inverse of the precision matrix $\boldsymbol{\Lambda}^{-1}$ is initialized to the covariance matrix $\mathbf{S}$ calculated using Eq. (1).

Repetition: The following processes are repeated until convergence as $i=1,2, \cdots, M, 1,2, \cdots$.

1) Create a new $(M-1) \times(M-1)$ matrix $\mathbf{W}$ by removing the $i$ th row and column from the inverse of the precision matrix $\Lambda^{-1}$ as follows:

$\boldsymbol{\Lambda}=\left(\begin{array}{cc}\mathbf{L} & \boldsymbol{l} \\ \boldsymbol{l}^{\mathrm{T}} & \lambda\end{array}\right), \boldsymbol{\Lambda}^{-1}=\left(\begin{array}{cc}\mathbf{W} & \boldsymbol{w} \\ \boldsymbol{W}^{\mathrm{T}} & \sigma\end{array}\right), \mathbf{S}=\left(\begin{array}{cc}\mathbf{R} & \boldsymbol{s} \\ \boldsymbol{s}^{\mathrm{T}} & s_{i, i}\end{array}\right)$

2) Solve the problem of lasso regression as follows:

$\min _{\boldsymbol{\beta}}\left\{\frac{1}{2}\left\|\mathbf{W}^{\frac{1}{2}} \boldsymbol{\beta}-\boldsymbol{b}\right\|^{2}+\rho\|\boldsymbol{\beta}\|_{1}\right\}$.

3) Replace the element $(i, i)$ of $\boldsymbol{\Lambda}^{-1}$ to $\sigma$ calculated using Eq. (4). The other elements of the $i$ th row and column of $\boldsymbol{\Lambda}^{-1}$ are replaced with $\boldsymbol{w}$ calculated using Eq. (5).

$\sigma=s_{i, i}+\rho$

$\boldsymbol{w}=\mathbf{W} \boldsymbol{\beta}$. 
4) Replace the element $(i, i)$ of $\boldsymbol{\Lambda}$ to $\lambda$ calculated using Eq. (6). The other elements of the $i$ th row and column of $\boldsymbol{\Lambda}$ are replaced with $\boldsymbol{l}$ calculated using Eq. (7).

$$
\begin{aligned}
& \lambda=\frac{1}{\sigma-\boldsymbol{w}^{\mathrm{T}} \mathbf{W}^{-1} \boldsymbol{w}}=\frac{1}{\sigma-\boldsymbol{\beta}^{\mathrm{T}} \mathbf{W}^{-1} \boldsymbol{\beta}} \\
& \boldsymbol{l}=-\frac{\mathbf{W}^{-1} w}{\sigma-\mathbf{w}^{\mathrm{T}} \mathbf{W}^{-1} \boldsymbol{W}}=-\frac{\boldsymbol{\beta}}{\sigma-\boldsymbol{\beta}^{T} \mathbf{W}^{-1} \boldsymbol{\beta}}
\end{aligned}
$$

Output the converged matrices $\boldsymbol{\Lambda}$ and $\boldsymbol{\Lambda}^{-1}$.

\subsection{Calculation of anomaly score using graphical lasso}

Ide et al. (2009) proposed a calculation method that quantifies the extent to which each variable contributes to the difference between $\mathcal{D}_{A}$ and $\mathcal{D}_{B}$ in terms of the variables themselves. Given the probabilistic models, the most natural difference measure is the Kullback-Leibler divergence, given as

$d_{i}^{\mathrm{AB}} \equiv \int \mathrm{d} z_{i} p_{\mathrm{A}}\left(z_{i}\right) \int \mathrm{d} x_{i} p_{\mathrm{A}}\left(x_{i} \mid z_{i}\right) \ln \frac{p_{\mathrm{A}}\left(x_{i} \mid z_{i}\right)}{p_{\mathrm{B}}\left(x_{i} \mid z_{i}\right)}$

This is the expected Kullback-Leibler divergence between $p_{\mathrm{A}}\left(x_{i} \mid z_{i}\right)$ and $p_{\mathrm{B}}\left(x_{i} \mid \boldsymbol{z}_{i}\right)$, integrated over the distribution $p_{\mathrm{A}}\left(z_{i}\right)$. By replacing $\mathrm{A}$ with $\mathrm{B}$ in Eq. (8), we obtain the definition of $d_{i}^{\mathrm{BA}}$. Because we assume this measure as a Gaussian, the integral can be analytically performed as

$d_{i}^{\mathrm{AB}}=\boldsymbol{w}_{\mathrm{A}}^{\mathrm{T}}\left(\boldsymbol{l}_{\mathrm{B}}-\boldsymbol{l}_{\mathrm{A}}\right)+\frac{1}{2}\left\{\frac{\boldsymbol{l}_{\mathrm{B}}^{\mathrm{T}} \mathbf{W}_{\mathrm{A}} \boldsymbol{l}_{\mathrm{B}}}{\lambda_{\mathrm{B}}}-\frac{\boldsymbol{l}_{\mathrm{A}}^{\mathrm{T}} \mathbf{W}_{\mathrm{A}} \boldsymbol{l}_{\mathrm{A}}}{\lambda_{\mathrm{A}}}\right\}+\frac{1}{2}\left\{\ln \frac{\lambda_{\mathrm{A}}}{\lambda_{\mathrm{B}}}+\sigma_{\mathrm{A}}\left(\lambda_{\mathrm{B}}-\lambda_{\mathrm{A}}\right)\right\}$,

where $\boldsymbol{\Lambda}_{\mathrm{A}}$ and its inverse $\boldsymbol{\Sigma}_{\mathrm{A}}$ are partitioned as

$\boldsymbol{\Lambda}_{\mathrm{A}}=\left(\begin{array}{cc}\mathbf{L}_{\mathrm{A}} & \boldsymbol{l}_{\mathrm{A}} \\ \boldsymbol{l}_{\mathrm{A}}^{\mathrm{T}} & \lambda_{\mathrm{A}}\end{array}\right), \boldsymbol{\Sigma}_{\mathrm{A}} \equiv \boldsymbol{\Lambda}_{\mathrm{A}}^{-1}=\left(\begin{array}{cc}\mathbf{W}_{\mathrm{A}} & \boldsymbol{w}_{\mathrm{A}} \\ \boldsymbol{w}_{\mathrm{A}}^{\mathrm{T}} & \sigma_{\mathrm{A}}\end{array}\right)$

respectively. A similar partitioning is also applied to $\boldsymbol{\Lambda}_{\mathrm{B}}$ and $\boldsymbol{\Sigma}_{\mathrm{B}}$. The expression for $d_{i}^{\mathrm{BA}}$ is then obtained by replacing A with $\mathrm{B}$ in the above equations.

By definition of the Gaussian graphical model, the number of nonzero entries in $\boldsymbol{l}_{\mathrm{A}}$ is the same as the degree of the node $x_{i}$. In this sense, $\boldsymbol{l}_{\mathrm{A}}$ contains information on the neighborhood graph of $x_{i}$. Therefore, the first term mainly detects the change in degree. The second term corresponds to the difference in the "tightness" of the neighborhood graph; specifically, if $x_{i}$ has a single link to $j$, then this term is proportional to the difference between the corresponding correlation coefficient normalized by the single-variable precisions $\lambda_{\mathrm{A}}$ and $\lambda_{\mathrm{B}}$. The third term is related to the change in the single-variable precisions (or variances). Here, $d_{i}^{A B}$ and $d_{i}^{B A}$ are quantities that indicate changes in the neighborhood graph of the $i$-th node. The greater the magnitudes of these quantities, the greater are the changes associated with the variable $x_{i}$. Thus, given the assumption of neighborhood preservation, Ide et al. (2009) reported that it is reasonable to define the anomaly score of the $i$-th variable as

$a_{i} \equiv \max \left\{d_{i}^{A B}, d_{i}^{B A}\right\}$

\section{Experimental design}

\subsection{Participants}

Eleven healthy, right-handed male students without any self-reported upper limb disorders participated in the experiment. All participants provided written informed consent after being briefed about the research protocol, which follows the principles outlined in the Declaration of Helsinki and was approved by the Research Safety Ethics Committee of Tokyo Metropolitan University. The mean \pm standard deviation values of the participants' ages, heights, and weights were $23.1 \pm 1.5$ years, $1,732 \pm 42 \mathrm{~mm}$, and $67.4 \pm 9.0 \mathrm{~kg}$, respectively. The mean \pm standard deviation 
values of the participants' hand lengths, forearm lengths, and upper limb lengths were $185 \pm 11,441 \pm 17$, and $740 \pm 27$ $\mathrm{mm}$, respectively.

\subsection{Experimental conditions and procedures}

In the experiment, the participants sat on chairs and moved 50 lightweight ball-shaped objects $($ diameter $=35 \mathrm{~mm}$, weight $=8 \mathrm{~g}$ ) under each task and each work-speed condition. The conditions included two types of tasks: Task A and Task B. In Task A, the participants moved the objects from a basket on their left to another basket on their right, whereas in Task B, the participants moved the objects from a basket placed farther away to another basket nearby. Participants were instructed to perform the lightweight material-handling tasks under three working speeds - specifically, fast, regular, and slow. The task intervals in fast, regular, and slow working speed conditions were set to $1,1.5$, and 2 s., respectively.

The participants were instructed to sit in a chair and maintain a steady anatomical position at the beginning of the experiment. After maintaining this position for $5 \mathrm{~s}$, the participants were requested to move the objects to the instructed baskets at the specified work speeds. All participants repeated the experimental procedures for all experimental conditions. The working pace was controlled with guidance from a metronome. The participants were instructed to maintain a rhythm of one movement for each work pace by matching the sound of the metronome to the moment at which the lightweight objects are picked up. After finishing the lightweight material-handling tasks, the participants maintained their anatomical positions again for $5 \mathrm{~s}$.

\subsection{Measured data and analysis}

\subsubsection{Working posture measurement}

An electromagnetic motion-tracking system (trakSTAR, Ascension Technology) was used to obtain the three-dimensional positions and orientations of the lower back, chest, right upper arm, right forearm, and right hand of the participants at a sampling frequency of $50 \mathrm{~Hz}$. The receiver placements for this data acquisition were selected to avoid soft-tissue artifacts, as referenced in Bourvier et al. (2015).

The joint angles of the trunk and right upper limb were calculated using the measured orientations of the body segments in three-dimensional rigid-link models. Then, the following anatomical joint angles were calculated: trunk inclination, trunk right inclination, trunk left rotation, shoulder abduction, shoulder flexion, shoulder inner rotation, elbow flexion, forearm pronation, wrist radial deviation, and wrist palmar flexion.

\subsubsection{Extraction of principal interjoint coordination using graphical lasso}

For each of the experimental conditions, the covariance matrices for all tasks were calculated. Subsequently, individual components of the covariance matrices were transformed to their absolute values, and the median values of each of these components were calculated for all participants. Similar processing was applied to the precision matrices estimated by graphical lasso as well. In this study, the regularization coefficient was set to 0.6. The diagonal components of the precision matrices estimated by the graphical lasso were ignored in this study because the focus was on the changes in the relationships with other joint angles.

\subsubsection{Estimation of the time-varying anomaly score}

To evaluate the effects of changes in the individual joint angles on the time-varying interjoint coordination, the anomaly scores in the time series were calculated according to the method in Ide et al. (2009). Specifically, the sequential working posture data were transformed into sub-sequence time series using a sliding window with a length of 250 frames. The first sub-sequence time series was defined as the reference data, and the anomaly scores of all the sub-sequence time-series data were calculated and compared with the reference data. Subsequently, the median anomaly scores were calculated for the eleven participants under each of the experimental conditions, and the calculated median anomaly scores were smoothed using exponential smoothing for a smoothing coefficient of 0.01 .

\section{Results}

\subsection{Example of joint angle variation}

Figure 1 shows the example of time-varying joint angles under fast work pace in Task A for one subject. The trunk left rotation, shoulder abduction, shoulder flexion, shoulder inner rotation, elbow flexion, and forearm pronation 
increased while picking-up the lightweight material and decreased while placing-down the lightweight material. In contrast, the wrist radial deviation and wrist palmar flexion angle decreased while picking-up the lightweight material and increased while placing-down the lightweight material. The trunk right inclination angle decreased slightly with the elapsed time. The amplitude of some joint angles, including trunk left rotation, shoulder abduction, shoulder flexion, and elbow flexion angle increased with the elapsed time. In contrast, the amplitude of the shoulder inner rotation angle decreased with the elapsed time. The examples of characteristic change in joint angle are shown in Figure 2 and Figure 3. Figure 2 shows time-varying shoulder flexion and shoulder inner rotation angle under regular work pace condition in Task A for one subject. The shoulder flexion angle changed in phase with the shoulder inner rotation angle. Figure 3 shows time-varying shoulder abduction and elbow flexion angle under regular work pace condition in Task B for one subject. The shoulder abduction angle changed in phase with the elbow flexion angle.
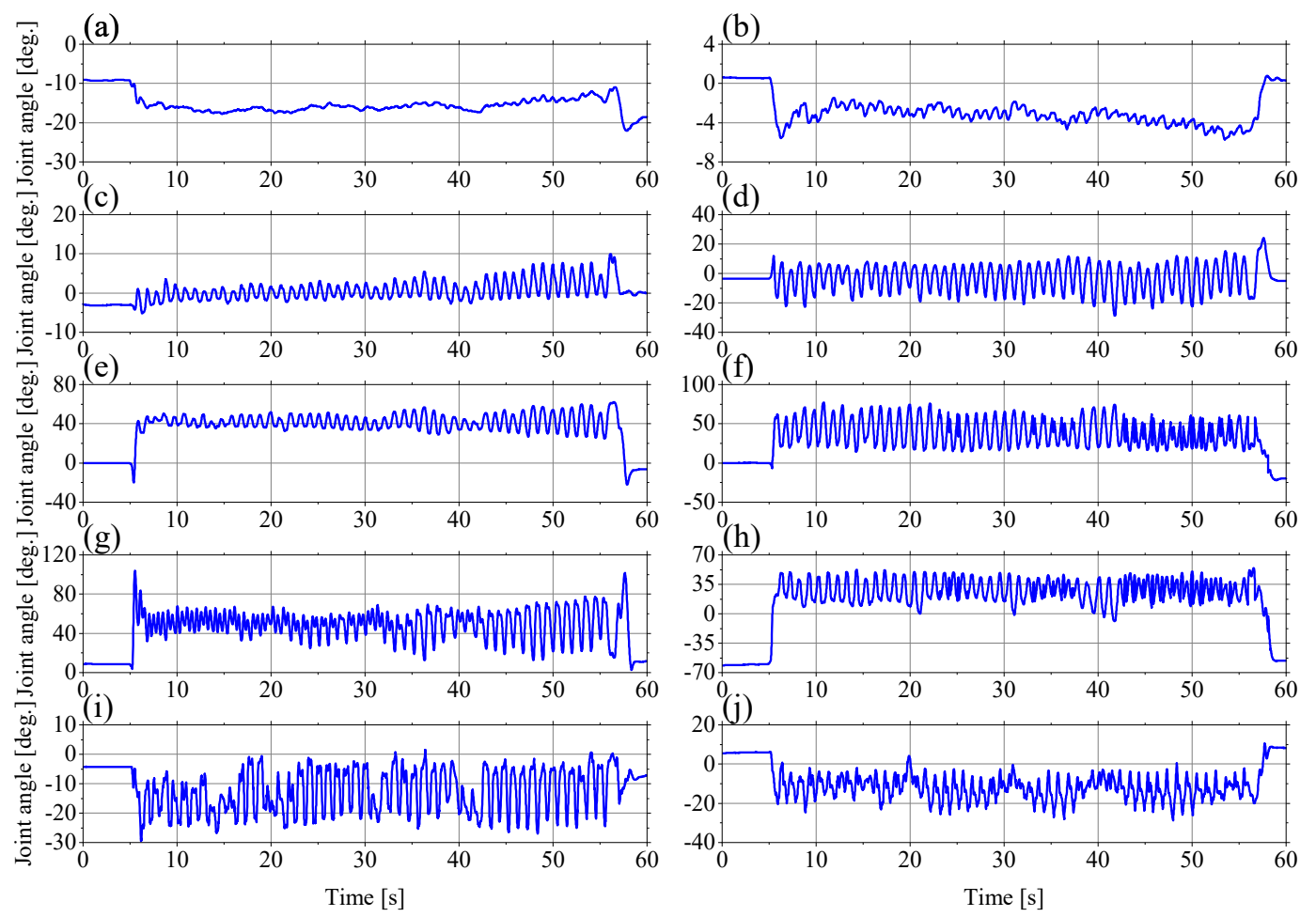

Fig. 1 Example of time-varying joint angles under fast work pace in Task A for one subject: (a) trunk inclination angle, (b) trunk right inclination angle, (c) trunk left rotation angle, (d) shoulder abduction angle, (e) shoulder flexion angle, (f) shoulder inner rotation angle, (g) elbow flexion angle, (h) forearm pronation angle, (i) wrist radial deviation angle, and (j) wrist palmar flexion angle. The trunk left rotation, shoulder abduction, shoulder flexion, shoulder inner rotation, elbow flexion, and forearm pronation increased while picking-up the lightweight material and decreased while placing-down lightweight material. In contrast, the wrist radial deviation and wrist palmar flexion angle decreased while picking-up the lightweight material and increased while placing-down the 


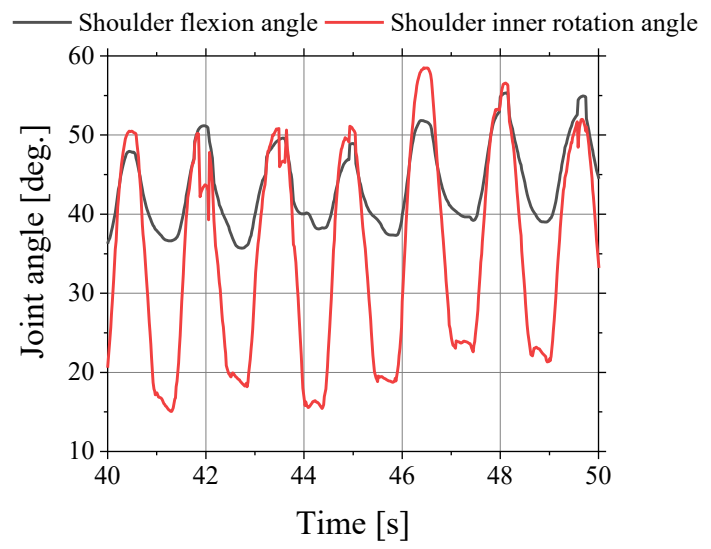

Fig. 2 Time-varying shoulder flexion and shoulder inner rotation angle under regular work pace condition in Task A for one subject. The shoulder flexion angle changed in phase with the shoulder inner rotation angle.

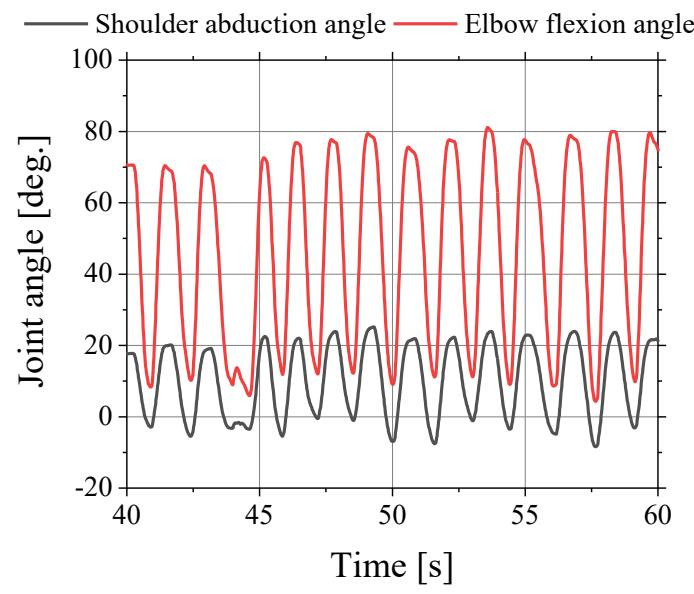

Fig. 3 Time-varying shoulder abduction and elbow flexion angle under regular work pace condition in Task B for one subject. The shoulder abduction angle changed in phase with the elbow flexion angle.

\subsection{Ensemble mean of each joint angle with normalized time}

Figure 4 and 5 present the variation in the ensemble mean of each joint angle with normalized time under each work pace in Task A and Task B, respectively. The sequential motion of picking-up a lightweight material and placing it down was normalized based on the ongoing pickup action and the subsequent one. Subsequently, the ensemble mean of each joint angle was calculated for all the participants. For each task condition, the trends of the joint angle that was most affected by the work pace condition or that exhibited the largest difference between the maximum and minimum angles were focused in this study.

In Task A, the participants assumed the reaching posture by significantly inclining the trunk to the left under the fast work pace condition than under other work pace conditions, as shown in Fig. 4(b). The average of the difference between the maximum and minimum angles in shoulder inner rotation for the three work pace conditions is approximately $35^{\circ}$, larger than those of the other joints.

In Task B, the trunk left rotation angle under a regular work pace condition was larger than that under other work pace conditions. The average of the difference between the maximum and minimum angles in elbow flexion for the three work pace conditions is approximately $51^{\circ}$, larger than those of the other joints. 

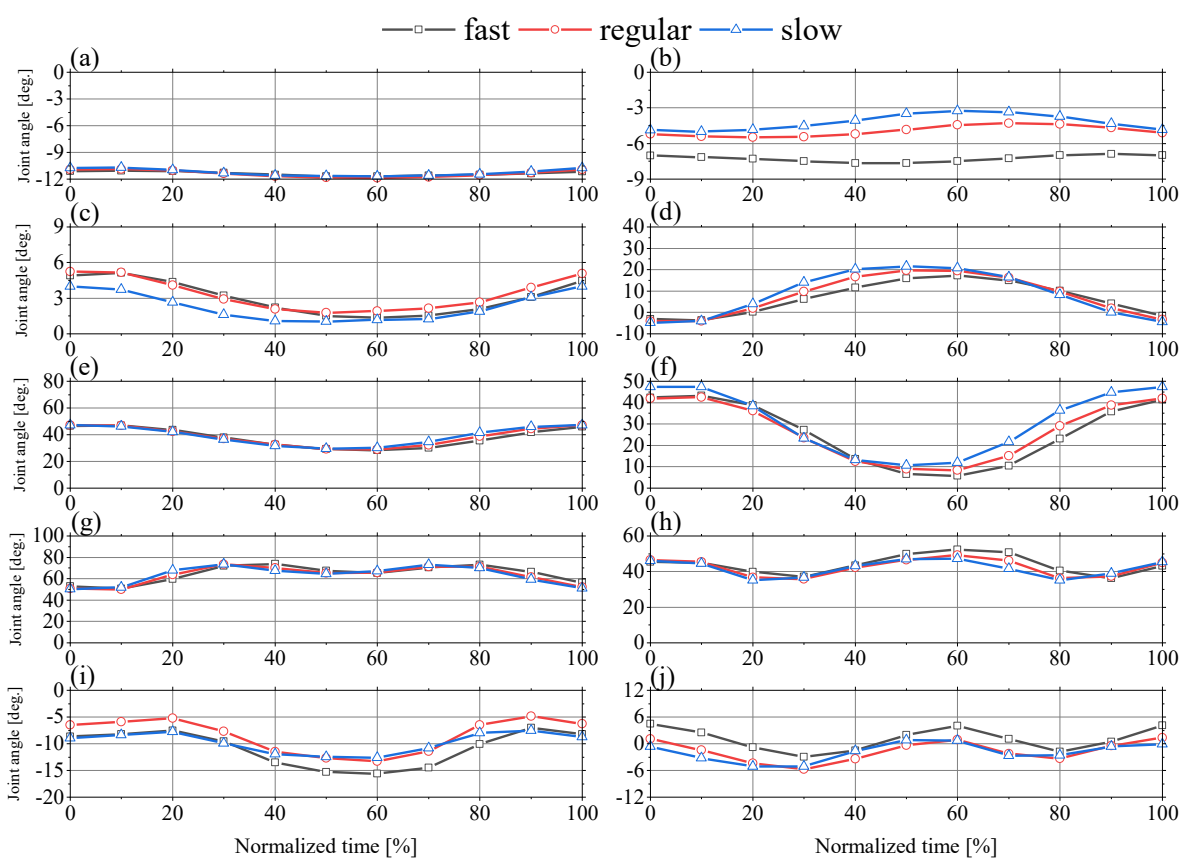

Fig. 4 Ensemble means of each joint angle as a function of normalized time under each work pace in Task A: (a) trunk inclination angle, (b) trunk right inclination angle, (c) trunk left rotation angle, (d) shoulder abduction angle, (e) shoulder flexion angle, (f) shoulder inner rotation angle, (g) elbow flexion angle, (h) forearm pronation angle, (i) wrist radial deviation angle, and (j) wrist palmar flexion angle. The participants assumed the reaching posture with significantly higher inclination of the trunk to the left under a fast work pace condition compared to other work pace conditions. The average of the difference between the maximum and minimum angle in shoulder inner rotation for the work pace conditions is approximately $35^{\circ}$, larger than those of the other joints.
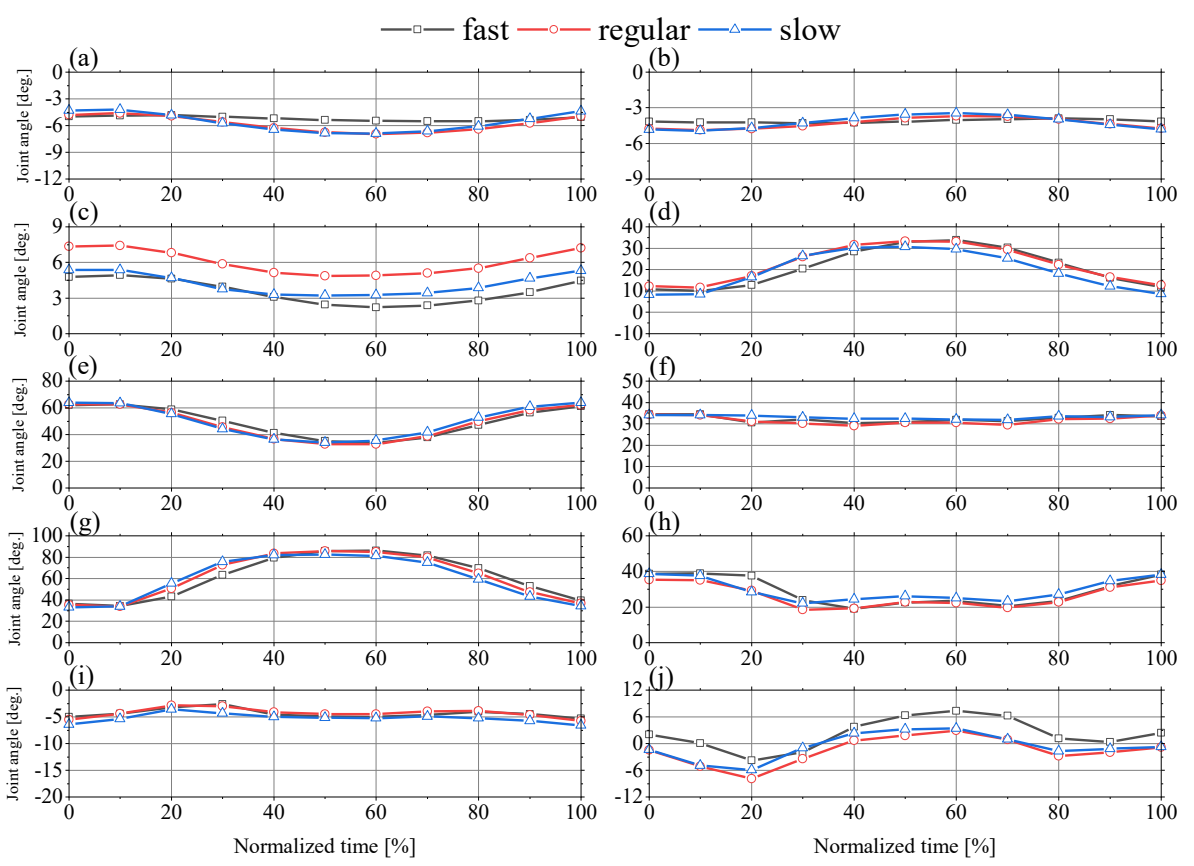

Fig. 5 Ensemble means of each joint angle as a function of normalized time under each work pace in Task B: (a) trunk inclination angle, (b) trunk right inclination angle, (c) trunk left rotation angle, (d) shoulder abduction angle, (e) shoulder flexion angle, (f) shoulder inner rotation angle, (g) elbow flexion angle, (h) forearm pronation angle, (i) wrist radial deviation angle, and (j) wrist palmar flexion angle. In Task B, the trunk left rotation angle under a regular work pace condition was larger than those under other work pace conditions. The average of the difference between the maximum and minimum angle in elbow flexion for the work pace conditions is approximately $51^{\circ}$, larger than those of the other joints. 


\subsection{Extraction of principal interjoint coordination using graphical lasso}

Figure 6 show the heat maps of the absolute values on the covariance matrix and on the precision matrix estimated by the graphical lasso under each experimental condition. According to the heat map of the precision matrix, it was shown that the precision matrix estimated by the graphical lasso enabled extraction of the principal interjoint coordination by comparison with the heat map of the covariance matrix. In Task A, the direct correlation between the trunk right inclination and forearm pronation angle was the largest under the fast work pace. For the regular or slow work pace condition, the direct correlation between the shoulder flexion and shoulder inner rotation angle was the largest. In Task B, the direct correlation between the shoulder abduction and elbow flexion angle was the largest for all working speeds.

\subsection{Estimation of the time-varying anomaly scores}

Figure 7 shows the time-varying anomaly score for each joint angle in Task A. The final anomaly score for the elbow joint angle in all experimental conditions was larger than those of the other joint angles, excluding that in the fast condition, where the final anomaly score for the wrist radial deviation angle was the largest.

Figure 8 shows the time-varying anomaly score for each joint angle in Task B. In all the experimental conditions, the final anomaly score on the forearm pronation angle was larger than those at the other joint angles.
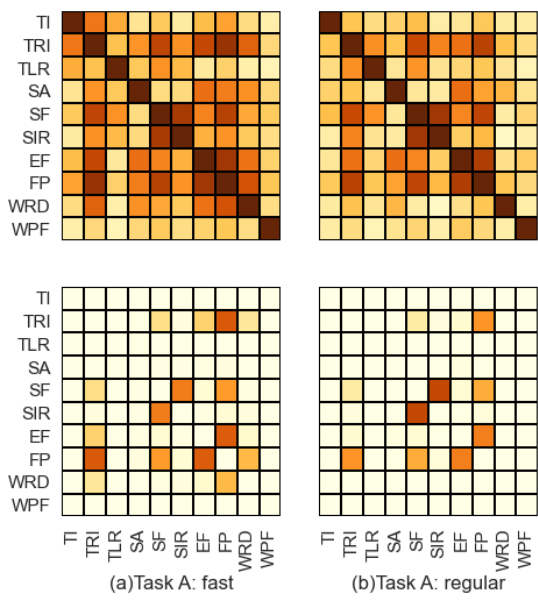
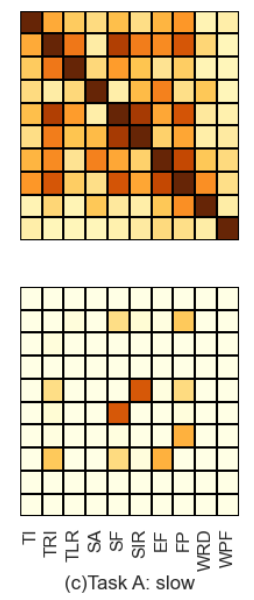
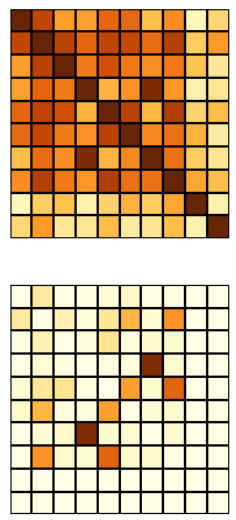

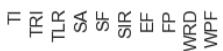

(d)Task B: fast
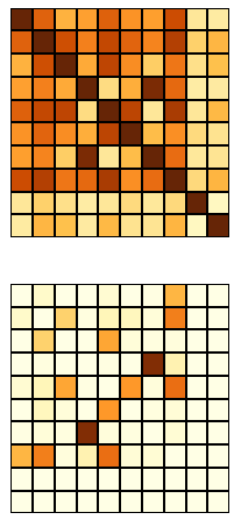

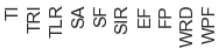

(e)Task B: regular
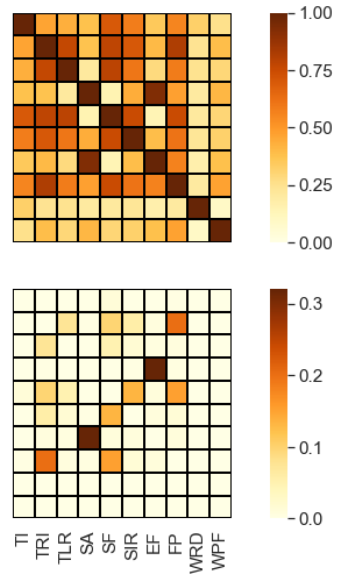

(f)Task B: slow

Fig. 6 Heat map of the absolute values of the covariance matrix and of the precision matrix estimated by the graphical lasso for each experimental condition (top: covariance matrix, bottom: precision matrix). The definitions of the joint angles in the figure are as follows: TI: trunk inclination angle, TRI: trunk right inclination angle, TLR: trunk left rotation angle, SA: shoulder abduction angle, SF: shoulder flexion angle, SIR: shoulder inner rotation angle, EF: elbow flexion angle, FP: forearm pronation angle, WRD: wrist radial deviation angle, and WPF: wrist palmar flexion angle. The precision matrix estimated by the graphical lasso enabled extraction of the principal interjoint coordination by comparison with the heat map of the covariance matrix. In Task A, the direct correlation between the trunk right inclination and forearm pronation angle was the largest under the fast work pace. For the regular or slow work pace condition, the direct correlation between the shoulder flexion and shoulder inner rotation angle was the largest. In Task B, the direct correlation between the shoulder abduction and elbow flexion angle was the largest for all working speeds. 


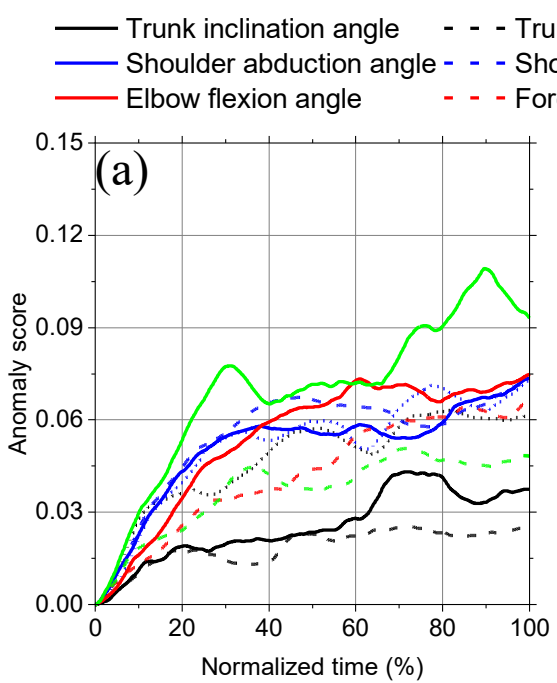

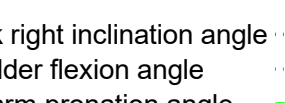

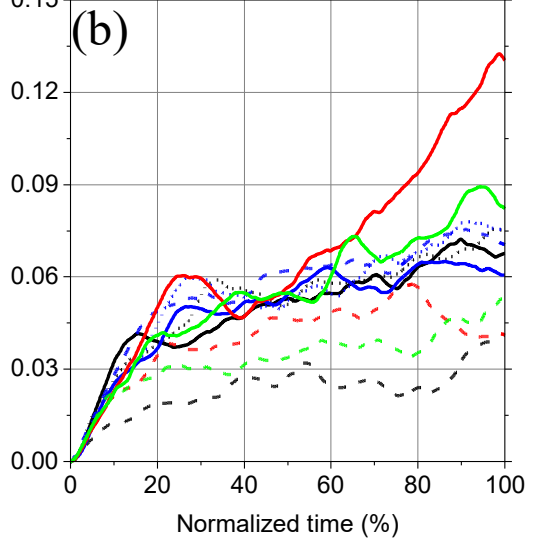

Trunk left rotation angle Shoulder inner rotation angle

Wrist radial deviation angle - - - Wrist palmar flexion angle

Fig. 7 Time-varying anomaly score of each joint angle in Task A: (a) fast, (b) regular, and (c) slow conditions. The final

anomaly score for the elbow joint angle in all experimental conditions was larger than those of the other joint
angles, excluding that in the fast condition, where the final anomaly score for the wrist radial deviation angle was

anomaly score for the elbow joint angle in all experimental conditions was larger than those of the other joint
angles, excluding that in the fast condition, where the final anomaly score for the wrist radial deviation angle was the largest.

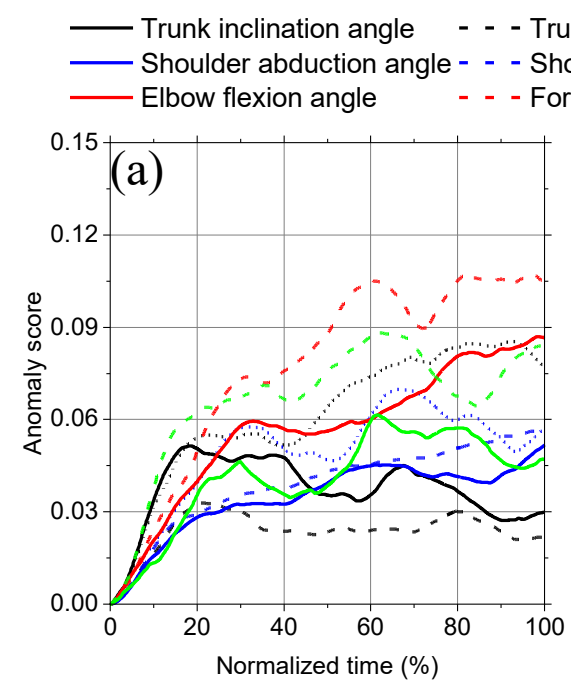

k right inclination angle
............

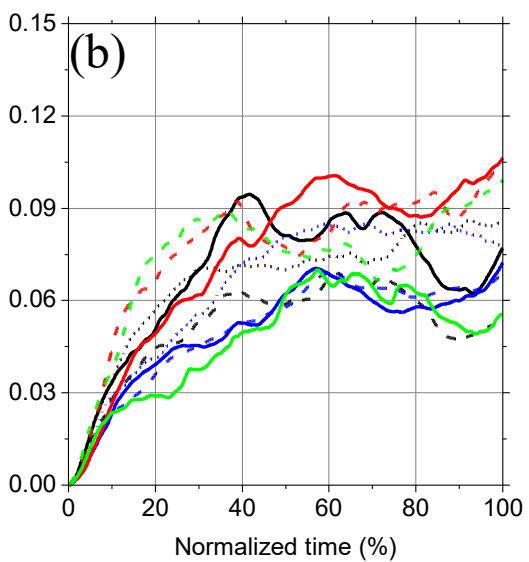

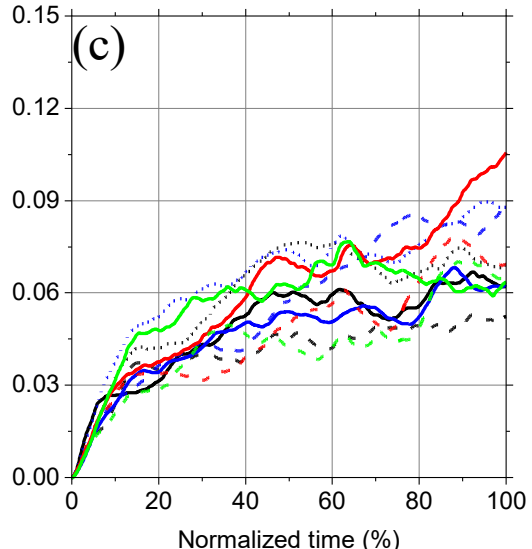

Fig. 8 Time-varying anomaly score of each joint angle in Task B: (a) fast, (b) regular, and (c) slow conditions. In all the experimental conditions, the final anomaly score on the forearm pronation angle was larger than those at the other joint angles.

\subsection{Trend of each term in the calculated anomaly score}

Figure 9 shows the time-varying anomaly scores for the elbow flexion angle in Task A and the time variation of each term in the calculation of the score. The results of the anomaly score show that the final score under fast work pace condition was smaller than those under the regular and slow work pace conditions. The trends of the anomaly scores were similar to those of the changes in the degree and single-variable precisions but differed from those of the tightness values of the neighborhood graphs.

Figure 10 shows the time-varying anomaly scores for the forearm pronation angle in Task B and the time variations of each of its terms. The results of the anomaly score show that the final score under fast work pace condition was smaller than those under the regular and slow work pace conditions. The trends of the anomaly scores were similar to those of the changes in the degree. However, the trends of the anomaly score for normalized time of $60 \%$ differed from those of the changes in the single-variable precisions. The trends of the anomaly scores also differed from those of the tightness values of the neighborhood graphs. 

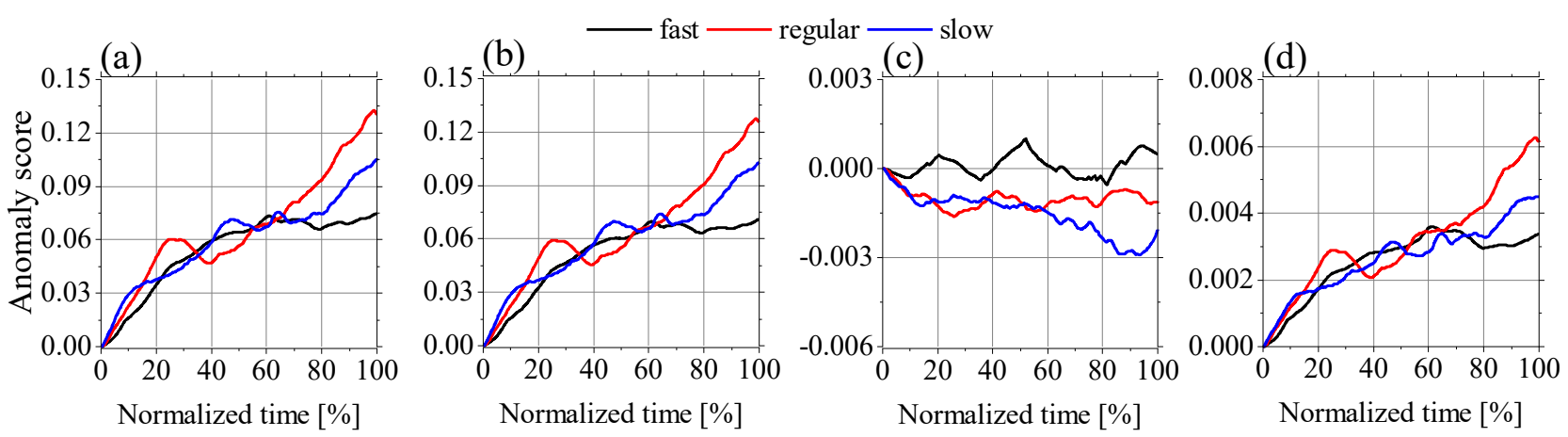

Fig. 9 Time-varying anomaly scores for the elbow flexion angle in Task A and time variation of each term in the calculation of the score: (a) anomaly score, (b) change in degree (first term in the anomaly score calculation), (c) tightness of the neighborhood graph (second term in the anomaly score calculation), and (d) changes in single-variable precisions (third term in the anomaly score calculation). The final anomaly score under fast work pace condition was smaller than those under the regular and slow work pace conditions. The trends of the anomaly scores were similar to those of the changes in the degree and single-variable precisions but differed from those of the tightness values of the neighborhood graphs.
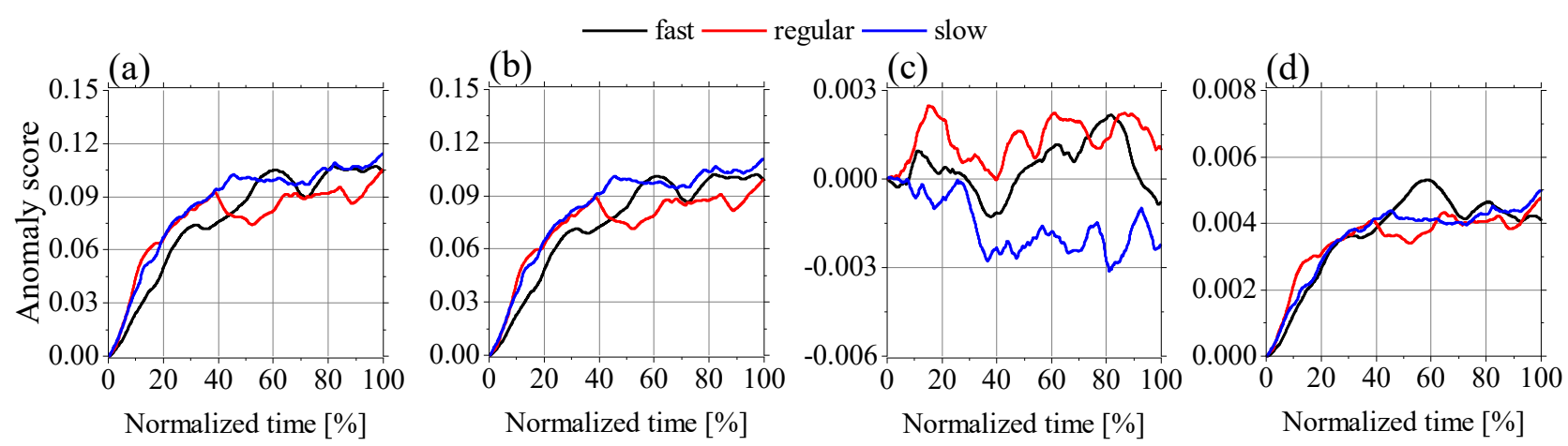

Fig. 10 Time-varying anomaly scores for the forearm pronation angle in Task B and time variation of each term in the calculation of the score: (a) anomaly score, (b) change in degree (first term in the anomaly score calculation), (c) tightness of the neighborhood graph (second term in the anomaly score calculation), and (d) changes in single-variable precisions (third term in the anomaly score calculation). The final anomaly score under fast work pace condition was smaller than those under the regular and slow work pace conditions. The trends of the anomaly scores were similar to those of the changes in the degree. However, the trends of the anomaly score for normalized time of $60 \%$ differed from those of the changes in the single-variable precisions. The trends of the anomaly scores also differed from those of the tightness values of the neighborhood graphs.

\section{Discussion}

In Task A, the direct correlation between the trunk right inclination and forearm pronation angle was largest under the fast work-speed condition and that between the shoulder flexion and inner rotation angle was largest under the regular and slow work-speed conditions. In Task B, the direct correlation between the shoulder abduction and elbow flexion angle was largest under all experimental conditions. In Task A, as the participants were forced to assume the reaching posture across the midline of the body, they elevated their right upper limbs by changing the shoulder flexion angles to avoid contact between the elbow joint and trunk during the lightweight material-handling tasks. Subsequently, to reach the basket on their left side, they rotated their shoulder joints inward; the shoulder flexion angle changed in phase with the shoulder inner rotation angle as shown in Figure 2. The results of the direct correlations in the regular and slow conditions under Task A suggest that the participants adopted the above movement strategy. However, the increase in the direct correlation between the trunk right inclination and forearm pronation angle suggests that the participants adopted different movement strategies to reach the basket more quickly. Specifically, to decrease the movement distance of the right upper limb, the participants assumed the reaching posture by inclining the trunk to the 
left as shown in Figure 4 (b). Therefore, the results under the fast work-speed conditions suggest that the direct correlation between the trunk right inclination and forearm pronation angle increased by adopting the above movement strategy and forearm pronation with the reaching movement. In Task B, the participants were instructed to move the lightweight materials from a basket placed farther away to another basket placed nearby. Yang et al. (2018) reported that the shoulder abduction angles changed in phase with elbow flexion angles during repetitive pointing tasks between the distal and proximal targets. In other words, they reported interjoint coordination between shoulder abduction and elbow flexion angle; the shoulder abduction angle changed in phase with the elbow flexion angle as shown in Figure 3. The extracted results of the principal interjoint coordination under Task B in this study are in agreement with the findings of Yang et al. (2018). The results of the extracted principal interjoint coordination activities in Task A and Task B suggested that the graphical lasso was suitable for extracting the characteristic interjoint coordination from human motion in the complete task.

In Task A, the final anomaly score of the elbow flexion angle was larger than those of the other joint angles under all experimental conditions, excluding that under the fast condition. This result suggests that the change in elbow flexion angle contributes to the difference between interjoint coordination at the onset and termination of the task. Similarly, in Task B, the final anomaly score of the forearm pronation angle was larger than those of the other joint angles under all experimental conditions, and this suggests that the change in this angle contributed to the difference between interjoint coordination at the onset and termination of the task. The final anomaly scores of the elbow flexion angle in Task A and that of the forearm pronation angle in Task B, shown in Figures 5 and 6, were smallest for the fast work-speed conditions. Srinivasan et al. (2015) reported that the movement variability decreased with increase in the working pace during repetitive tasks. Therefore, as the difference between the working posture or motion at the onset and termination of the task reduced by decreased movement variability during the repetitive lightweight material-handling task, the final anomaly score in the fast work-speed condition was smaller than those in the other work-speed conditions. However, the final anomaly score of the elbow flexion angle under the slow work-speed condition was smaller than that under the regular work-speed conditions.

In the work pace experiments where the participants were allowed to work at their comfortable pace, movement variability increased by easy changes to the working postures. However, movement variability decreased in the experimental conditions that forced the work pace. The result of the time-varying anomaly score of elbow flexion angle under Task A indicated that the trend of anomaly score was similar to the changes in the degree and single-variable precisions. This result suggested that the elbow joint actively changed in task A and influenced the difference between interjoint coordination at the onset and termination of the task. Conversely, the result of the time-varying anomaly score of the forearm pronation angle under Task B indicated that the trend of the anomaly score was similar to the change in the degree but was not similar to the change in the single-variable precision from the normalized time of $60 \%$. This result suggested that the forearm contributed the most to the difference between interjoint coordination at the onset and termination of the task but passively varied with the changes in the other joints. These findings suggest that the anomaly score enables extraction of the principal joint angles that influence the differences between interjoint coordination at the onset and termination of tasks. Moreover, analyzing the changes in each term of the anomaly score calculation might determine whether the joint angles actively changed during the tasks.

The experimental conditions in this study had several limitations. Note that the results obtained in this study were biased to the right-handed male participants. Srinivasan et al. (2016) reported that women and men did not differ in the ability to perform repetitive tasks at low-to-moderate force levels but that the movement strategies employed in repetitive tasks differed by gender. Therefore, if an experiment targeting female participants was conducted, the interjoint coordination and movement strategies may differ from the results in this study. In Task A, the participants moved the objects from a basket on their left to another basket on their right by the dominant hand. When the left-handed participants performed Task A, the interjoint coordination and movement strategies may differ from that of right-handed participants. In this study, the number of participants was relatively small, and only young adults participated. The individual differences such as age, gender, physical size, and dominant hand might have affected the change of interjoint coordination and movement strategies in repetitive tasks. Therefore, the influence of these characteristics should be investigated in subsequent studies with significantly more participants. 


\section{Conclusion}

To evaluate tasks that have low physical workloads or small variations in the working postures, an evaluation method based on human motion characteristics is required. The interjoint coordination, which produces multijoint movements by organizing the redundant DoFs into fewer major covarying relationships, is one of the primary characteristics of human motion. This study validated the efficacy of the graphical lasso tool for evaluation of interjoint coordination and working postures in time-series data under repetitive lightweight material handling, with changes in the task type and work-speed conditions. The estimated precision matrix using the graphical lasso suggests that the principal interjoint coordination can be extracted and reflects the differences in movement strategies for each task type in comparison with the covariance matrix. The results of the time-varying anomaly scores suggest that the joint angle that contributes most to the difference between interjoint coordination during task onset and termination can be determined from the final anomaly score. Moreover, the analysis of time variations of each term of the anomaly score calculation suggests that the active or passive effects of joint angles on interjoint coordination in time-series data can be determined. The effect of individual differences such as age, gender, physical size, and dominant hand on the inter-joint coordination and movement strategies should be investigated in subsequent studies with significantly more participants.

\section{Acknowledgement}

This work was supported by JSPS KAKENHI Grant Number 19K04922.

\section{References}

Bernstein, N., The Coordination and Regulation of Movements (1967), Pergamon Press: Oxford.

Bouvier, B., Duprey, S. and Dumas, R., Upper limb kinematics using inertial and magnetic sensors: Comparison of sensor-to-segment calibrations, Sensors, Vol.15, No.8 (2015), pp.18813-18833.

Chaffin, D. B., Andersson, G. B. J. and Martin, B. J., Occupational Biomechanics $4^{\text {th }}$ ed. (2006), Wiley-Interscience.

Cowley, J. C. and Gates, D. H., Inter-joint coordination changes during and after muscle fatigue, Human Movement Science, Vol.56 (2017), pp.109-118.

Côté, J. N., Feldman A. G., Mathieu, P. A. and Levin, M. F., Effects of fatigue on intermuscular coordination during repetitive hammering, Motor Control, Vol.12, No.2 (2008), pp.79-92.

Côté, J. N., Mathieu, P. A., Levin, M. F. and Feldman, A. G., Movement reorganization to compensate for fatigue during sawing, Experimental Brain Research, Vol.146 (2002), pp.394-398.

Dempster, A. P., Covariance selection, Biometrics, Vol.28, No.1 (1972), pp.157-175.

Fantini, P., Tavola, G., Taisch, M., Barbosa, J., Leitao, P., Liu, Y., Sayed, M. S. and Lohse, N., Exploring the integration of the human as a flexibility factor in CPS enabled manufacturing environments: Methodology and results, Proceedings of the $42^{\text {nd }}$ Conference of the Industrial Electronics Society (IECON-2016) (2016), pp.5711-5716.

Farina, D., Leclerc, F., Arendt-Nielsen, L., Buttelli, O. and Madeleine, P., The changes in spatial distribution of upper trapezius muscle activity is correlated to contraction duration, Journal of Electromyography and Kinesiology, Vol.18, No.1 (2008), pp.16-25.

Friedman, J., Hastie, T. and Tibshirani, R., Sparse inverse covariance estimation with the graphical lasso, Biostatistics, Vol.9, No.3 (2008), pp.432-441.

Forsman, M., Hasson, G. Å., Medbo, L., Asterland, P. and Engström, T., A method for evaluation of manual work using synchronized video recordings and physiological measurements, Applied Ergonomics, Vol.33, No.6 (2002), pp.533-540.

Gates, D. H. and Dingwell, J. B., The effects of muscle fatigue and movement height on movement stability and variability, Experimental Brain Research, Vol.209 (2011), pp.525-536.

Huffenus, A. F., Amarantini, D. and Forestier, N., Effects of distal and proximal arm muscle fatigue on multi-joint movement organization, Experimental Brain Research, Vol.170 (2006), pp.438-447.

Ide, T., Lozano, A. C., Abe, N. and Liu, Y., Proximity-based anomaly detection using sparse structure learning, Society for Industrial and Applied Mathematics - Proceedings of the 2009 SIAM International Conference on Data Mining (2009), pp.96-107. 
Kadir, B. A., Briberg, O. and da Conceiçã, C. S., Current research and future perspective on human factors and ergonomics in Industry 4.0, Computers \& Industrial Engineering, Vol.137 (2019), DOI:10.1016/j.cie.2019.106004.

Kadrolkar, A. and Sup IV, F. C., Intent recognition of torso motion using wavelet transform feature extraction and linear discriminant analysis ensemble classification, Biomedical Signal Processing and Control, Vol.38 (2017), pp.250-264.

Karhu, O., Kansi, P. and Kuorinka, I., Correcting working posture in industry: A practical method for analysis, Applied Ergonomics, Vol.8, No.4 (1977), pp.199-201.

Li, Q., Qiao, M., Bian, W. and Tao, D., Conditional graphical lasso for multi-label image classification, Proceedings of the IEEE Conference on Computer Vision and Patten Recognition (2016), pp.2977-2986.

Masoud, H. I., Zerehsaz, Y. and Jin, J., Analysis of human motion variation patterns using UMPCA, Applied Ergonomics, Vol.59 (2017), pp.401-409.

McAtamney, L. and Corlett, E. N., RULA: A survey method for the investigation of work-related upper limb disorders, Applied Ergonomics, Vol.24, No.2 (1993), pp.91-99.

McDonald, A. C., Tse, C. T. F. and Keir, P. J., Adaptations to isolated shoulder fatigue during simulated repetitive work, Part II: Recovery, Journal of Electromyography and Kinesiology, Vol.29 (2016), pp.42-49.

San-Segundo, R., Montero, J. M., Barra-Chicote, R., Fernández, F. and Pardo, J. M., Feature extraction from smartphone inertial signals for human activity segmentation, Signal Processing, Vol.120 (2016), pp.359-372.

Srinivasan, D., Samani, A., Mathiassen, S. E. and Madeleine, P., The size of structure of arm movement variability decreased with work pace in a standardised repetitive precision task, Ergonomics, Vol.58, No.1 (2015), pp.128-139.

Srinivasan, D., Sinden, K. E., Mathiassen, S. E. and Côté, J. N., Gender differences in fatigability and muscle activity responses to a short-cycle repetitive task, European Journal of Applied Physiology, Vol.116 (2016), pp.2357-2365.

Sun, S., Huang, R. and Gao, Y., Network-scale traffic modeling and forecasting with graphical lasso and neural networks, Journal of Transportation Engineering, Vol.138, No.11 (2012), DOI:10.1061/(ASCE)TE.1943-5436.0000435.

Varrel, J., Pologe, S., Manselle, W., Lindenberger, U. and Woollacott, M., Coordination of degrees of freedom and stabilization of task variables in a complex motor skill: Expertise-related differences in cello bowing, Experimental Brain Research, Vol.224, No.3 (2013), pp.323-334.

Wang, X., O’Dwyer, N., Halaki, M. and Smith, R., Identifying coordinative structure using principal component analysis based on coherence derived from linear systems analysis, Journal of Motor Behavior, Vol.45, No.2 (2013), pp.167-179.

Yang, C., Bouffard, J., Srinivasan, D., Ghayourmanesh, S., Cantu, H., Begon, M. and Côté, J. N., Changes in movement variability and task performance during fatiguing repetitive pointing task, Journal of Biomechanics, Vol.76 (2018), pp.212-219. 